\title{
Exploiter les données du dossier médical informatisé pour améliorer la qualité des soins en ambulatoire
}

\author{
Pr ARNAUD CHIOLERO ${ }^{\mathrm{a}, \mathrm{b}}$, Dr JEAN-PAUL CALBIMONTE ${ }^{c}$, Dr GAETANO MANZO ${ }^{c}$, BRUNO ALVES $^{d}$, \\ Pr MICHAEL SCHUMACHER ${ }^{c}$, Dr SAMUEL GAILLARD ${ }^{d}$, Dr PHILIPPE SCHALLER ${ }^{e}$ et Pre VALÉRIE SANTSCHI ${ }^{\dagger}$
}

Rev Med Suisse 2021; 17: 2056-9

\begin{abstract}
Les professionnels de santé souhaitent des indicateurs pour monitorer la qualité des soins ambulatoires en exploitant au mieux les données récoltées de routine; la finalité est de fournir des soins de haute valeur, centrés sur le patient, fondés sur l'évidence et orientés par les données. Alors que cela semble simple de produire des indicateurs via le dossier médical informatisé (DMI), ces données ne parlent pas toutes seules. En effet, il faut: a) rendre les données exploitables; b) définir des indicateurs pertinents et $c$ ) assurer la diffusion de ces indicateurs auprès des patients et professionnels de santé. Dans cet article, nous explicitons comment le DMI peut être utilisé pour produire des indicateurs de qualité des soins ambulatoires en prenant l'exemple de l'hypertension et du diabète.
\end{abstract}

Leveraging data from electronic medical record to improve quality of outpatient care

Healthcare providers need indicators to monitor the quality of ambulatory care by making the best use of routinely collected data; the goal is to provide high-value, patient-centered, evidence-based, and data-informed health care. While it may seem simple to produce indicators via the electronic medical record (EMR), these data do not speak by themselves. Indeed, it is necessary to: a) make the data usable; $b$ ) define relevant indicators; and $c$ ) ensure the dissemination of these indicators to patients and healthcare providers. In this article, we explain how the EMR can be used to produce indicators of quality of ambulatory care, using the example of hypertension and diabetes.

\section{INTRODUCTION}

Monitorer la qualité des soins en ambulatoire est un élément essentiel pour l'améliorer.' Le rapport national sur la qualité des soins a mis en exergue la faiblesse des systèmes d'information existants pour monitorer la qualité des soins et produire des indicateurs pertinents en ambulatoire ${ }^{2,3}$ et la FMH en a aussi fait une priorité. ${ }^{4}$ Produire de tels indicateurs

aLaboratoire de santé des populations (\#PopHealthLab), Université de Fribourg, 1700 Fribourg, bobservatoire valaisan de la santé (OVS), 1970 Sion, 'Unité e-Health, Institut informatique de gestion, HES-SO Valais, 3960 Sierre, ' Logival SA, 3960 Sierre, ${ }^{e}$ Réseau Delta Romandie, 1213 Onex, Institut et haute école de la santé La Source, HES-SO Haute école spécialisée de Suisse occidentale, 1004 Lausanne

arnaud.chiolero@unifr.ch | jean-paul.calbimonte@hevs.ch

gaetano.manzo@hevs.ch | alves@logival.ch | michael.schumacher@hevs.ch gaillard@logival.ch | schaller@gmo.ch | v.santschi@ecolelasource.ch et intégrer leur emploi dans la pratique permettent la mise en œuvre d'un système de santé apprenant en continu (continuously learning health system), ${ }^{5}$ la prise de décision clinique aidée par les données (data-informed health care) et la fourniture de soins centrés sur la valeur (value-based health (are). ${ }^{6}$

Le dossier médical informatisé (DMI) est a priori idéal comme source de données pour un tel monitoring. ${ }^{7}$ En effet, des données cliniques dans le DMI sont récoltées de routine, en temps réel, et il peut dès lors paraître simple de produire des indicateurs de qualité à partir de ces données. Le potentiel est considérable, mais certaines difficultés souvent sous-estimées rendent difficiles la production et le suivi d'indicateurs. D'une part, une grande partie des données sont insuffisamment structurées pour être exploitables et, d'autre part, il manque une stratégie de production et de diffusion des indicateurs conçue avec les professionnels de santé (figure 1).

Dans cet article, nous discutons des conditions pour que le DMI puisse être utilisé pour produire des indicateurs de qualité des soins ambulatoires en prenant l'exemple de l'hypertension et du diabète.

\section{DOSSIER MÉDICAL INFORMATISÉ VS DOSSIER ÉLECTRONIQUE DU PATIENT}

En préambule, il est essentiel de distinguer le DMI du dossier électronique du patient (DEP). Le DMI (EMR en anglais pour «Electronic Medical Record») est la version électronique du dossier médical du patient dans une structure de soins. C'est un système dit «primaire» géré directement par le médecin, le pharmacien et l'équipe soignante qui travaillent dans la structure de soins. Le DMI contient des données cliniques brutes, plus ou moins structurées, du texte saisi librement et des documents de santé (ordonnance, rapport de sortie de l'hôpital, carnet de vaccination, radiographies). Mediway est un exemple de DMI utilisé par beaucoup de médecins en Suisse. ${ }^{8}$

Le DEP (PHR en anglais pour «Personal Health Record») est un dossier électronique en ligne qui appartient au patient. C'est un système dit «secondaire» qui contient des documents de santé tels que des ordonnances, rapports de sortie de l'hôpital, carnet de vaccination ou radiographies. ${ }^{9}$ Ces documents sont déposés par les professionnels de santé ou par le patient lui-même. Les droits d'accès sont gérés par le patient. En Suisse romande, l'association CARA propose un DEP. ${ }^{9}$ 
Processus et tâches en lien avec la production d'indicateurs de la qualité des soins à partir des données ambulatoires du dossier médical informatisé des patients.

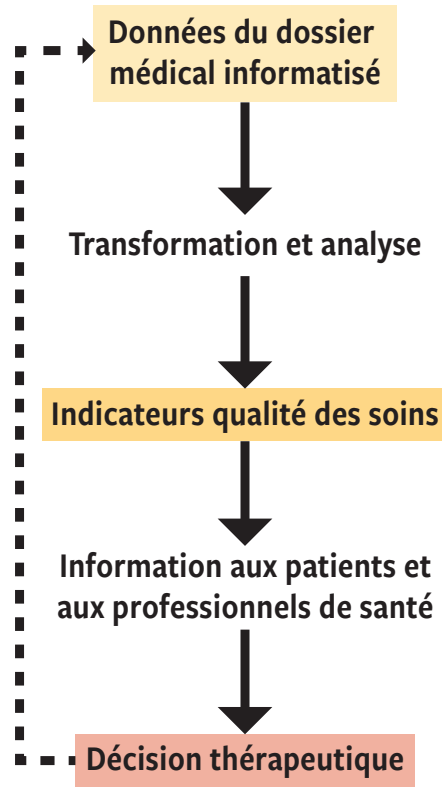

\section{Rendre les données exploitables \\ - S'assurer du droit d'accès, d'exploitation des données et de leur diffusion et informer le patient \\ - Aider la saisie et améliorer la structuration via des codifications et ontologies}

\section{Produire des indicateurs pertinents}

- Fondés sur l'évidence

- Validés par les professionnels de santé et les patients

\section{S'assurer que l'indicateur serve}

à la décision

- Processus clair de diffusion des indicateurs

- Accessibilité différenciée en fonction des besoins

\section{LES DONNÉES NE PARLENT PAS TOUTES SEULES}

Une erreur fréquente dans le monitoring est de croire que les données électroniques parlent facilement et qu'il suffit d'un bon outil d'exploitation de données. C'est insuffisant car les données ne parlent pas toutes seules.

Pour qu'un système d'informations basées sur des données électroniques de routine produise de l'information utile à la décision, il faut: a) rendre les données exploitables (garantir le droit d'exploitation et renforcer la structuration); b) définir des indicateurs pertinents et c) assurer la diffusion de ces indicateurs auprès des patients et professionnels de santé (figure 1). À toutes les étapes, il faut une collaboration étroite entre les usagers - médecins, pharmaciens, soignants et patients - et les experts en informatique médicale et science des données.

\section{Rendre les données exploitables}

D’une part, il faut s'assurer du droit d'exploiter ces données et dans quelle mesure il est possible de diffuser l'information qu'on en tire. Les données du DMI sont potentiellement utiles pour les soins aux patients, pour évaluer la qualité des soins et à des fins de recherche. Ce sont des données de santé personnelles et le patient doit être informé de leur exploitation à des fins d'amélioration de la qualité des soins. Un consentement explicite est nécessaire uniquement pour exploiter ces données à des fins de recherche.

D'autre part, il faut augmenter la qualité des données saisies via des interfaces faciles d'emploi pour les professionnels de la santé. ${ }^{10}$ Traditionnellement, la saisie se fait en texte libre, manuellement, et les données peuvent être très hétérogènes, avec certains termes ou abréviations employés différemment entre patients, d'une consultation à l'autre et entre professionnels de santé. Le processus peut être amélioré dès la saisie en transformant du texte libre en information structurée et par le traitement automatique de langage naturel. Des outils facilitent et automatisent la saisie et le codage des données par enrichissement sémantique via un vocabulaire normalisé (par exemple, en proposant à l'utilisateur des termes issus d'un dictionnaire de référence) ${ }^{11}$ (figure 2). La codification de l'information saisie est nécessaire et peut se faire via des standards de terminologies cliniques. L'utilisation de systèmes de classification et de codage de diagnostics, de procédures et traitements (tableau 1) est nécessaire pour ensuite appliquer des méthodes de prédiction, classification et profilage..$^{10}$

Par exemple, dans le cas du traitement de l'hypertension, atteindre une valeur de pression artérielle systolique considérée comme satisfaisante est un objectif de soins et la proportion de patients «contrôlés» est un indicateur (de résultat) de la qualité des soins ${ }^{12}$ (tableau 2). Pour produire cet indicateur, il faut, d'une part, prévoir un champ dans le DMI pour saisir les valeurs de pression et, d'autre part, définir explicitement ce que l'on considère comme étant une pression contrôlée.

\section{Définir des indicateurs pertinents}

On distingue trois types d'indicateurs, à savoir de structure, de processus et de résultat. Le choix initial des indicateurs peut être fait sur la base de recommandations de bonne pratique établies par des organismes experts, ${ }^{13}$ en tenant compte de la disponibilité et de la qualité des données dans le DMI. Un élément essentiel est d'impliquer les utilisateurs du DMI - médecins, pharmaciens, soignants - et les patients dans le choix de ces indicateurs, leur définition et leur opérationnalisation mais aussi dans comment cette information va circuler. 
Étapes dans la production d'indicateurs de la qualité des soins à partir des données du dossier médical informatisé MediWay (www.mediway.ch/). FHIR: Fast Healthcare Interoperability Resources (norme pour l'échange de données sur les soins de santé); ICPC: International Classification of Primary Care (en français CISP: Classification internationale des soins primaires).

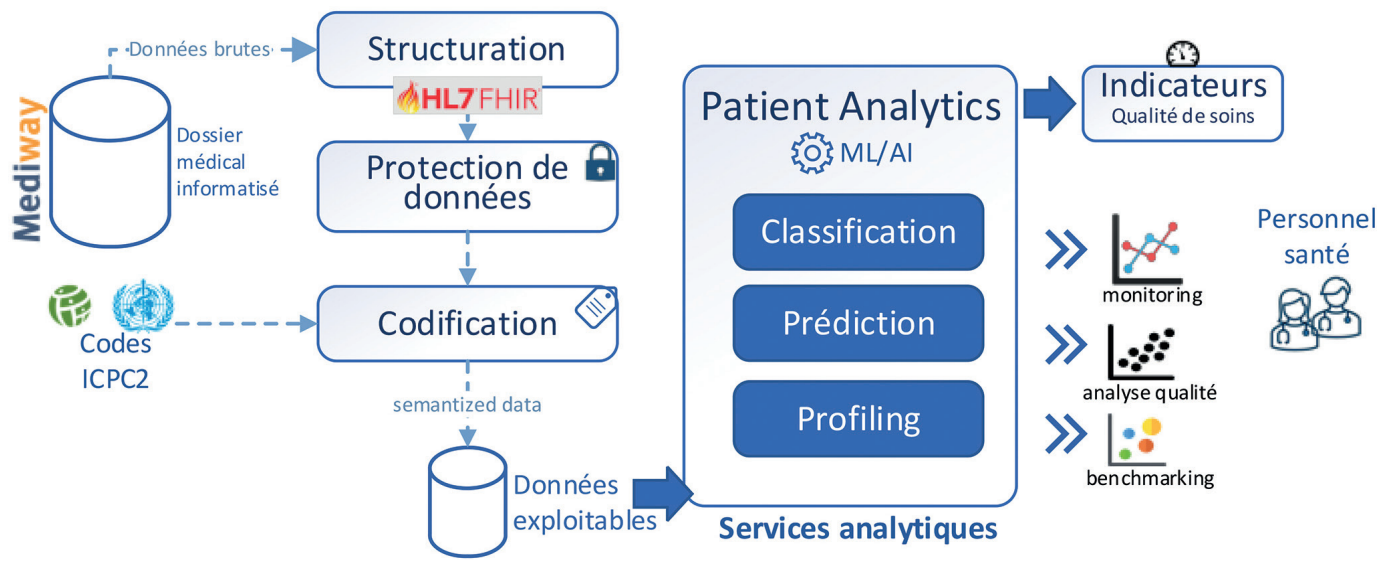

\section{TABLEAU}

\section{Aperçu de certaines terminologies cliniques utilisées dans les DMI}

a www.snomed.org/; b www.globalfamilydoctor.com/groups/WorkingParties/wicc.aspx; c www.who.int/classifications/icd/en/; d www.loinc.org/; e www.aapc.com/resources/ medical-coding/cpt.aspx; ${ }^{\dagger}$ www.whocc.no/; DMI : dossier médical informatisé.

\begin{tabular}{|c|c|c|c|}
\hline $\begin{array}{l}\text { Système de codage } \\
\text { (description) }\end{array}$ & Domaine d'application & Type & Objectif/usage \\
\hline $\begin{array}{l}\text { SNOMED-CT } \\
\text { (Systematized Nomenclature of Medicine - Clinical Terms) }\end{array}$ & $\begin{array}{l}\text { Plusieurs domaines (diagnostics, allergies, symp- } \\
\text { tômes, etc.) }\end{array}$ & Terminologie & Documentation clinique \\
\hline $\begin{array}{l}\text { ICPC }^{\mathrm{b}} \\
\text { (International Classification of Primary Care) }\end{array}$ & Diagnostic, motif de visite, procédures & Classification & Rapport, dossier clinique \\
\hline $\begin{array}{l}\text { ICD } \\
\text { (International Classification of Diseases) }\end{array}$ & Diagnostic & Classification & Rapport, dossier clinique \\
\hline $\begin{array}{l}\text { LOINC } \\
\text { (Logical Observation Identifiers Names and Codes) }\end{array}$ & Tests de laboratoire & Terminologie & Documentation clinique \\
\hline $\begin{array}{l}\text { CPT }^{\mathbf{e}} \\
\text { (Current Procedural Terminology) }\end{array}$ & Procédures & Terminologie & Documentation clinique \\
\hline $\begin{array}{l}\text { ATC }^{f} \\
\text { (Anatomical Therapeutic Chemical) }\end{array}$ & Médicaments & Classification & Rapport \\
\hline
\end{tabular}

Dans le cas du diabète, un exemple d'indicateur de processus est la proportion de patients avec diabète qui ont eu récemment un examen ophtalmologique du fond d'œil ${ }^{14}$ (tableau 2). Un exemple d'indicateur de résultat est la proportion de patients avec une hémoglobine glyquée considérée comme satisfaisante. Idéalement, le DMI devrait permettre d'identifier des patients particuliers où les standards de prise en charge ne s'appliquent pas. Le DMI est aussi potentiellement utile pour identifier des séquences d'action clinique (clinical action measures) telles que «résultat pas satisfaisant $\rightarrow$ intervention thérapeutique $\rightarrow$ meilleur résultat ou non $\rightarrow$...» et prévenir ainsi l'inertie clinique. ${ }^{14}$ Enfin, le DMI devrait permettre la saisie des expériences (PREMS: Patient-reported experience measures) et des résultats (PROMS: Patient-reported outcomes measures) de soins rapportés par les patients. ${ }^{15}$

\section{Assurer la diffusion des indicateurs}

Les modalités de diffusion des indicateurs doivent être pensées en fonction de leur usage, et ce dans le respect de la protection des données (figure 1). Il est utile de distinguer les usages suivants: a) aider à la prise en charge du patient (suivi historique et comparaison avec d'autres patients); b) faire du benchmarking (se situer par rapport à un objectif considéré comme optimal et se comparer avec d'autres pourvoyeurs de soins) et c) diffuser les résultats de manière anonyme (par exemple dans un rapport d'activité).

Ainsi, un médecin sera intéressé d'avoir accès à un indicateur qui résume où se situe un patient en comparaison avec d'autres patients similaires, et qui indique l'objectif à atteindre (pression artérielle systolique inférieure à $140 \mathrm{mmHg}$ ), ou qui rappelle lorsqu'un examen doit être réalisé (par exemple du fond d'œil). Sous cette forme, non anonyme, l'indicateur est utilisé uniquement par les personnes qui ont accès au DMI. Pour la comparaison entre pourvoyeurs de soins, les données doivent être anonymisées. Enfin, l'indicateur aura un format différent pour diffuser publiquement le résultat. Il faut organiser le retour aux pourvoyeurs de soins et s'assurer qu'ils ont accès aux résultats de ces indicateurs sous un format adéquat (figure 1). 


\begin{tabular}{|c|c|c|c|c|c|}
\hline & \multicolumn{2}{|c|}{ TABLEAU 2} & \multicolumn{2}{|c|}{$\begin{array}{c}\text { Indicateurs de la qualité } \\
\text { des soins }\end{array}$} & \\
\hline \multicolumn{6}{|c|}{$\begin{array}{l}\text { Le tableau ci-dessous propose des exemples d'indicateurs de la qualité des soins } \\
\text { dans la prise en charge de l'hypertension et du diabète. }{ }^{12} \\
\text { Les seuils pour définir ce qui est considéré comme un contrôle satisfaisant sont } \\
\text { discutés et personnalisés. } \\
\text { PA: pression artérielle. }\end{array}$} \\
\hline \multicolumn{2}{|l|}{ Indicateurs } & \multicolumn{2}{|c|}{$\begin{array}{l}\text { Type } \\
\text { d'indicateur }\end{array}$} & \multicolumn{2}{|l|}{ Définition } \\
\hline \multicolumn{6}{|l|}{ HTA } \\
\hline \multicolumn{2}{|c|}{ 1. Mesure de la PA } & \multicolumn{2}{|c|}{ Processus } & \multicolumn{2}{|c|}{$\begin{array}{l}\text { Mesure de la PA dans les } 12 \text { der- } \\
\text { niers mois }\end{array}$} \\
\hline \multicolumn{2}{|c|}{ 2. Traitée ou non } & \multicolumn{2}{|c|}{ Processus } & \multicolumn{2}{|c|}{$\begin{array}{l}\text { Prescription de médicament } \\
\text { antihypertenseur }\end{array}$} \\
\hline \multicolumn{2}{|c|}{$\begin{array}{l}\text { 3. Contrôle satisfaisant } \\
\text { de la PA }\end{array}$} & \multicolumn{2}{|c|}{ Résultat } & \multicolumn{2}{|c|}{ PA systolique $<130-160 \mathrm{mmHg}$} \\
\hline \multicolumn{6}{|l|}{ Diabète } \\
\hline \multicolumn{2}{|c|}{$\begin{array}{l}\text { 1. Autocontrôle } \\
\text { glycémique }\end{array}$} & \multicolumn{2}{|c|}{ Processus } & \multicolumn{2}{|c|}{ Fait des mesures de glycémie } \\
\hline \multicolumn{2}{|c|}{$\begin{array}{l}\text { 2. Contrôle glycémique } \\
\text { satisfaisant }\end{array}$} & \multicolumn{2}{|c|}{ Résultat } & \multicolumn{2}{|c|}{ Hémoglobine glyquée $<7-9 \%$} \\
\hline \multicolumn{2}{|c|}{ 3. Mesure de la PA } & \multicolumn{2}{|c|}{ Processus } & \multicolumn{2}{|c|}{$\begin{array}{l}\text { Mesure de la PA dans les } 12 \text { der- } \\
\text { niers mois }\end{array}$} \\
\hline \multicolumn{2}{|c|}{$\begin{array}{l}\text { 4. Contrôle satisfaisant } \\
\text { de la PA }\end{array}$} & \multicolumn{2}{|c|}{ Résultat } & \multicolumn{2}{|c|}{ PA systolique $<130-160 \mathrm{mmHg}$} \\
\hline \multicolumn{2}{|c|}{ 5. Examen du fond d'œil } & \multicolumn{2}{|c|}{ Processus } & \multicolumn{2}{|c|}{$\begin{array}{l}\text { Examen réalisé dans les } 12 \text { mois } \\
\text { précédents }\end{array}$} \\
\hline \multicolumn{2}{|c|}{ 6. Examen du pied } & \multicolumn{2}{|c|}{ Processus } & \multicolumn{2}{|c|}{$\begin{array}{l}\text { Examen réalisé dans les } 12 \text { mois } \\
\text { précédents }\end{array}$} \\
\hline
\end{tabular}

\section{CONCLUSION}

Les données du DMI ont un potentiel immense pour le monitoring de la qualité des soins en ambulatoire..$^{14,16}$ Toutefois, leur exploitation nécessite une véritable stratégie de monitoring, allant de la saisie de la donnée à la diffusion de l'information aux cliniciens. Le but est d'avoir de l'information utile, d'une part, pour la prise en charge du patient et, d'autre part, pour l'amélioration en continu des processus et systèmes de soins. C'est la clé pour aller vers des soins ambulatoires de haute valeur, centrés sur le patient, fondés sur l'évidence et guidés par les données. ${ }^{6}$

Conflit d'intérêts: Le Dr Gaillard et M. Alves sont actifs au sein de Logival qui produit le DMI MediWay. Les autres auteurs n'ont déclaré aucun conflit d'intérêts en relation avec cet article.

Remerciements: Au Dr Jean-Gabriel Piguet, Institut d'informatique de gestion, Sierre, pour ses suggestions en lien avec la protection et le droit d'exploitation des données.

\section{IMPLICATIONS PRATIOUES}

- Les professionnels de santé souhaitent des indicateurs pour monitorer la qualité des soins ambulatoires en exploitant au mieux les données récoltées de routine via le dossier médical informatisé (DMI)

- Toutefois, ces données ne parlent pas toutes seules

- Pour que les données du DMI soient utiles pour le monitoring de la qualité des soins, il faut: a) rendre ces données exploitables; b) définir des indicateurs pertinents et $c$ ) définir un processus de diffusion de ces indicateurs
1 Agency for Healthcare Research and Quality (AHRQ). The Challenge and Potential for Assuring Quality Health Care for the 21st Century. Disponible sur: www.ahrq.gov/patient-safety/qualitymeasures/21st-century/index.html. 2 *Office fédéral de la santé publique. Développement de la qualité des soins en Suisse. Disponible sur : www.bag.admin. $\mathrm{ch} /$ bag/fr/home/versicherungen/ krankenversicherung/developpementqualite-en-suisse.htm

3 Chiolero A, Rodondi N. Quality Improvement in Primary Care: Toward the Provision of Safe, High Value, Patient-Centered, Sustainable, and Data-Informed Care. Swiss National Report on Quality and Safety in Health- care. 2019. Disponible sur : https://boris. unibe.ch/135693/

4 Fédération des médecins suisses.

Qualité/ASQM. Disponible sur: www.fmh. $\mathrm{ch} / \mathrm{fr} /$ themes/qualite-asqm.cfm

5 McGinnis JM, Fineberg HV, Dzau VJ.

Advancing the Learning Health System. N Engl J Med 2021;385:1-5.

6 Chiolero A, Rodondi N, Santschi V. High-Value, Data-Informed and TeamBased Care for Multimorbidity. Lancet Public Health 2020;5:e84.

7 Romano MJ, Stafford RS. Electronic Health Records and Clinical Decision Support Systems: Impact on National Ambulatory Care Quality. Arch Intern Med 2011:171:897-903.

8 Mediway. www.mediway.ch/
9 CARA. www.cara.ch/fr/Public.html. 10 Gopinath D, Agrawal M, Murray L, et al. Fast, Structured Clinical Documentation via Contextual Autocomplete. Proc Mach Learn Res 2020;106:1-25.

11 Haendel MA, Chute CG, Robinson PN. Classification, Ontology, and Precision Medicine. N Engl J Med 2018;379:1452-62.

12 Santschi V, et al. Team-Based Care for Improving Hypertension Management among Outpatients (TBC-HTA): Study Protocol for a Pragmatic Randomized Controlled Trial. BMC Cardiovasc Disord 2017;17:39.

13 *Calsbeek $\mathrm{H}$, et al. Performance Measurements in Diabetes Care: The Complex Task of Selecting Quality
Indicators. Int J Qual Health Care 2013:25:704-9.

14 *0'Connor PJ, et al. Diabetes Performance Measures: Current Status and Future Directions. Diabetes Care 2011;34:1651-9.

15 Makhni EC, Swantek AJ, Ziedas AC, et al. The Benefits of Capturing PROMs in the EMR. NEJM Catal Innov Care Deliv 2021;2:10.1056/CAT.21.0134. Disponible sur : https://catalyst.nejm.org/doi/ full/10.1056/CAT.21.0134

16 Friedman DJ, Parrish RG. The

Population Health Record: Concepts, Definition, Design, and Implementation. J Am Med Inform Assoc 2010;17:359-66.

* à lire 